\title{
London 2012 Olympic and Paralympic Opening and Closing Ceremonies: Audience Pixel Content
}

\author{
Ed Cookson and Will Case \\ Crystal CG \\ 31-35 Kirby Street \\ London, EC1N 8TE \\ edcookson@me.com,will@fizzymilk.com
}

\section{INTRODUCTION}

While the Opening and Closing Ceremonies of each successive Olympic Games have tended to grow increasingly sophisticated in their scope and scale, a key ambition of the London 2012 Ceremonies team was to integrate the in-stadium audience as part of the show, emphasising the narrative themes of inclusiveness and togetherness. Furthermore, the large size of an Olympic stadium can make it harder to create an intimate and immersive live experience for the stadium audience. In response, 70,500 audience seats in the stadium were fitted with a 9-LED pixel paddle, all of which were wired to a central visual controller. The result was an enormous immersive display that holds the current world-record for the largest landscape video display. Live equipment company Tait Technology designed and installed the tablets, known as the Audience Pixels, while software designers Immersive provided the $37 \mathrm{Ai}$ Servers that controlled the LEDs. Digital media studio Crystal CG created all 14 hours of Audience Pixels content for the Olympic and Paralympic Opening and Closing Ceremonies, and an intensive period of creative and technical $R \& D$ was required to get the best out of the innovative display.

\section{EXPOSITION}

The production and implementation of the visual content required close collaboration with the teams of Creative Directors, AV Producers, Technicians, Stage Designers and Video Directors assembled by the London 2012 Organising Committee for each Ceremony - particularly 59 Productions, Treatment Studio, Dan Sloane, Dave Bartlett and the LOCOG Ceremonies AV team lead by Justine Catterall. To create the motion graphics content, Crystal CG assembled a production team comprised of over 40 Motion Graphic Designers, Animators, 3D Designers and Producers. An additional 54 dedicated 8-core blades were added to their existing high specification Render Farm, to cope with round-the-clock rendering requirements, and also the changing briefs as the shows evolved through rehearsals and iterative integration of the digital content into the narrative of each ceremony. Due to the unique size, shape and nature of the display, Crystal CG faced numerous visualisation challenges.

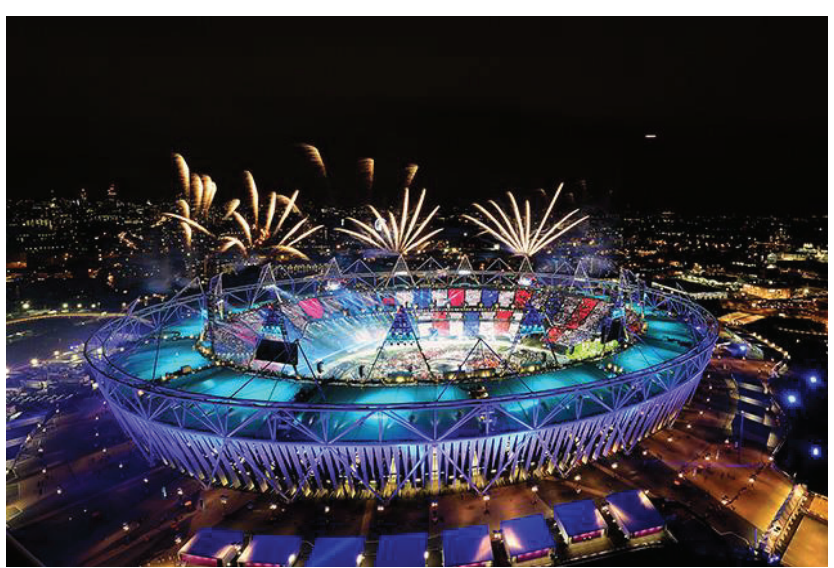

Figure 1: London 2012 Olympics Opening Ceremony (Getty Images)

\subsection{Visualisation Challenges}

The first few months of 2012 were spent trying to understand how the panels would display seemingly simple creative ideas. Circles would not appear circular, certain colours would appear washed out, and it took a while to get the speeds of objects moving around the stadium to "feel right". The time available to view creative ideas on the actual Audience Pixels themselves was also restricted, due to installation timescales and limited Stadium access. While the simulation of ideas in 
the studio was possible using Immersive's $\mathrm{Ai}$ software, it took a while to understand the relationship between how results would appear in the visualisation software and how they would look and feel in the stadium.

\section{RESULTS}

The resulting immersive experience extended the visual canvas from the field of play up into the stadium audience, making them part of the show and enhancing the broadcast spectacle. A video of edited highlights of the Audience Pixels as featured in the four Ceremonies is available online here: http://vimeo.com/48434161

The Ceremonies in general were well received by both critics and the public. As hoped, the Audience Pixels appear to have been a hit with the instadium audiences, as well as the broadcast audience (estimated at around $900 \mathrm{~m}$ worldwide for the Olympics Opening Ceremony).

\section{CONCLUSIONS}

While production for the Audience Pixels posed many creative, technical and logistical challenges, the results of their use in the four London 2012 Opening and Closing Olympic and Paralympic Ceremonies points to an evolution of the presentation of digital content in live events, creating an ever more immersive experience. For the ceremonies, the technology and content were designed entirely around a cohesive creative narrative. While there are numerous learnings from the challenges faced in London 2012 that can help to deliver future events, results are likely to be more successful if they are led by a similarly strong event narrative, rather than applying the technology for its own sake.

www.crystalcg.co.uk 